\title{
République et relations entre les peuples
}

Quelques éléments de l'idéal républicain autour de brumaire an VIII

Hervé Leuwers

\section{(2) OpenEdition}

Journals

Édition électronique

URL : https://journals.openedition.org/ahrf/292

DOI : $10.4000 / a h r f .292$

ISSN : 1952-403X

Éditeur :

Armand Colin, Société des études robespierristes

Édition imprimée

Date de publication : 1 décembre 1999

Pagination : 677-693

ISSN : 0003-4436

Référence électronique

Hervé Leuwers, "République et relations entre les peuples », Annales historiques de la Révolution française [En ligne], 318 | octobre-décembre 1999, mis en ligne le 11 avril 2006, consulté le 24 avril 2022. URL : http://journals.openedition.org/ahrf/292 ; DOI : https://doi.org/10.4000/ahrf.292

Ce document a été généré automatiquement le 24 avril 2022.

Tous droits réservés 


\title{
République et relations entre les peuples
}

\author{
Quelques éléments de l'idéal républicain autour de brumaire an VIII
}

\section{Hervé Leuwers}

1 Dans les mois qui précédèrent et qui suivirent le 18 Brumaire, l'emblème de la République restait une femme, souvent casquée, à côté de laquelle était fréquemment représentée une pique portant bonnet phrygien, symbole de liberté. La fidélité à une image, que la montée d'un pouvoir personnel et autoritaire devait progressivement émousser ${ }^{1}$, était le signe d'un attachement proclamé à des " principes » à la hauteur desquels, selon Garat, les responsables politiques devaient veiller à toujours se hisser ${ }^{2}$; ces derniers reconnaissaient l'existence d'un idéal républicain vers lequel il fallait tendre ; ils croyaient que la République, pour reprendre des propos de Claude Nicolet, s'incarnait dans "une doctrine, ou un esprit [...] toujours en devenir " ${ }^{3}$. Dans les discours des partisans de la Révolution, la République, celle des derniers mois du Directoire et des premiers du Consulat, se reconnaissait dans des images et des valeurs qui définissaient une véritable exception française.

2 C'est l'une des dimensions de cette identité républicaine que cet article tentera de mettre en évidence. L'idéal "révolutionnaire ", tel qu'il s'incarne dans la liberté faite femme ${ }^{4}$, contient une nouvelle conception des relations entre les peuples que T.C.W. Blanning présente comme un élément central d'une culture politique régénérée ${ }^{5}$; en donnant à cette notion de culture politique une acception plus restreinte ${ }^{6}$, on peut souligner que la définition de ces nouveaux rapports ne fut ni unique, ni immuable : en fonction des périodes et des mouvances politiques considérées, cet idéal a pu prendre des formes diverses. En l'an VIII et en l'an IX, pendant ce moment clef qui voit la fin de la Révolution proclamée et la guerre se poursuivre contre la seconde coalition, tous les Français n'en ont évidemment pas la même conception; pour autant, la presse officielle, l'édition ou les débats des assemblées propagent une vision dominante qui paraît occulter toute conception dissonante ; c'est cette image que nous rechercherons dans les débats qui suivirent l'assassinat des plénipotentiaires français à Rastadt (floréal an VII), la crise gouvernementale du 30 Prairial, la relance des négociations de 
paix par Bonaparte (nivôse an VIII), l'affaire Blackwell ou les succès des armées françaises en Italie du Nord au printemps 1800.

Inévitablement, cette recherche d'un idéal républicain dominant sera sous-tendue par plusieurs questions : celle de l'importance, immédiate ou différée, de la rupture du 18 Brumaire dans son élaboration ou sa transformation; celle de la permanence éventuelle d'une utopie planétaire, d'un rêve d'une république universelle ou d'une fraternité entre tous les peuples ${ }^{7}$; celle des relations entretenues avec les pratiques ou les écrits de l'Ancien Régime et des différentes assemblées révolutionnaires. L'image de l'idéal républicain, telle qu'elle ressort de cette analyse, apparaît riche de multiples contradictions, qui n'entament pas la conviction affichée des hommes de l'an VIII de défendre, avec force et sincérité, une conception profondément renouvelée des rapports entre les peuples.

La France, gardienne du droit des gens

4 L'idée d'une nécessaire régulation et moralisation des rapports entre les peuples, développée par nombre de juristes et de philosophes des XVII et $\mathrm{XVIII}^{\mathrm{e}}$ siècles, et défendue par l'Assemblée constituante, avait acquis la force d'un principe. En l'an VIII et en l'an IX, ponctuellement, le rappel de l'existence d'un droit des gens, supérieur aux lois des États et garant de relations policées entre les gouvernements et les peuples, de même que la représentation de la République comme gardienne de ses règles, démontraient que la loyauté envers une éthique des relations entre peuples était considérée comme partie intégrante de l'idéal républicain.

5 Le droit des gens, également désigné par l'expression "droit des nations " ${ }^{8}$, était présenté comme un ensemble de "règles d'équité et d'humanité " ${ }^{9}$, comme un corpus de principes qui formait un « fragile et respectable frein, qui modère dans leurs fureurs les nations belligérantes ${ }^{10}$; ces règles, qui ne concernaient pas exclusivement le droit de la guerre, interdisaient l'empoisonnement des fontaines, les violences militaires envers les femmes, les vieillards et les enfants, le respect des ambassadeurs et des citoyens d'une nation étrangère. Reconnues comme autant d'obligations éthiques, dont on attribuait la formulation à " cette foule de bons écrits qui [avaient] illustré le xviII siècle ${ }^{11}$, elles paraissaient généralement devoir s'imposer à l'humanité entière.

Évidemment, la définition de ce droit des gens variait ; à la manière des philosophes et des juristes de leur siècle, les auteurs pouvaient notamment se diviser sur l'analyse de son origine. Dans le débat sur les sources du droit des gens, deux grandes écoles s'opposaient : pour Pufendorf et ses partisans, il n'existait pas de droit des gens positif car ses préceptes procédaient d'un droit naturel universel et immuable; pour Bynkershoek ou Moser, au contraire, ces règles étaient le produit de la volonté des États et se définissaient par l'étude des traités et des usages. Entre les convictions de Pufendorf et celles de Bynkershoek, pour reprendre deux hommes qui s'opposaient, la part laissée au droit naturel dans la présentation des sources du droit des gens pouvait varier à l'infini ${ }^{12}$. Ces différences d'analyse se retrouvent dans la définition du droit des nations livrée au public ; pourtant, lorsque l'origine de ce droit est présentée, c'est la référence au droit naturel qui l'emporte de très loin, il est vrai de manière laconique et peu explicite: en floréal an VII, dans leur dénonciation de l'assassinat des plénipotentiaires français Roberjot et Bonnier, attribué aux ordres du gouvernement autrichien, les directeurs ainsi que le ministre François de Neufchâteau assimilaient en tout ou partie droit des gens et droit naturel ${ }^{13}$, tandis que Benoist, un an plus tard, assurait que « nulle loi positive » n'avait constitué ce droit qui avait « régi de tout tems 
les querelles des grands peuples " ${ }^{14}$. Il ne nous appartient pas ici de disserter sur l'origine philosophique ou l'attachement des locuteurs à ces références ${ }^{15}$; sans nous éloigner de l'observation des discours, il nous suffira de constater qu'en l'an VIII encore, la référence au droit naturel, comme élément fondateur du droit des gens, demeurait d'usage courant.

7 La remarque est d'importance et paraît confirmée par la portée universelle que la plupart des auteurs attribuaient au droit des nations. Certes, une fois encore, les propos relevés peuvent différer. Dans leur message du 16 floréal an VII (5 mai 1799), les directeurs considéraient le droit des gens en usage chez les " peuples civilisés " ${ }^{16}$; bien que le laconisme de l'expression doive inciter à la prudence, l'on peut remarquer que la pensée de Martens, à la veille de la Révolution, sans totalement nier la force du droit naturel, notamment en cas de silence du droit positif, voulait considérer les règles du droit des gens comme positives et uniquement applicables à des États disposant d'un même degré de civilisation ${ }^{17}$. Quelle que soit la conviction des rédacteurs du message du directoire exécutif, l'on doit souligner que l'image d'un droit des gens de portée universelle domine largement dans les textes; pour Jean Debry, il s'impose même aux " nations les plus barbares " ${ }^{18}$; chez Heurtault-Lamerville, il s'assimile à une « morale universelle ${ }^{19}$; pour Bailleul, il tisse entre les peuples des liens étroits et réciproques qui permettent de préserver le « contrat social » ou le « pacte » qui fonde l'humanité ${ }^{20}$.

Les références fréquentes au droit naturel et à l'humanité, même en l'absence de définition précise des mots ou de renvoi explicite aux philosophes ou aux jusnaturalistes modernes, permettaient de préserver l'image de l'universalisme du droit des nations, dont le respect intéressait non seulement la France, mais aussi l'ensemble des hommes. Le respect de ses règles devenait dès lors un enjeu fondamental, souligné par l'adjectif «sacré », fréquemment apposé à l'expression droit des gens ${ }^{21}$. Dans les débats qui suivirent l'assassinat des plénipotentiaires français à Rastadt, l'atteinte faite à l'immunité diplomatique était présentée comme le viol d'un droit constitutif de l'ordre mondial, comme la dissolution du contrat qui unissait les différents peuples de la terre dans une même communauté humaine. Bailleul aux CinqCents et Gourdan aux Anciens expliquaient que ce crime avait rompu le "pacte qui unissait les nations par le droit des gens » et isolé les peuples les uns des autres ${ }^{22}$; ce "parricide des nations", pour reprendre une expression d'Heurtault-Lamerville ${ }^{23}$, semblait faire de la France la « seule nation » (Bailleul) ${ }^{24}$ et suspendre temporairement les droits des autres peuples. Par leurs crimes, les Autrichiens se trouvaient rejetés de l'humanité ; ils devenaient des «monstres", des «cannibales", des «sauvages", des "barbares" ${ }^{25}$... Ils n'étaient plus des hommes, et l'Autriche cessait d'être "une puissance ${ }^{26}$. Quant au gouvernement de ce pays, ses crimes présumés en faisaient "l'irréconciliable ennemi des nations. " ${ }^{27}$

Dans les discours, l'image de l'idéal républicain se contruisait par référence au contremodèle autrichien. Lors de l'affaire de Rastadt, à l'époque des offres de paix de Bonaparte à l'Angleterre et à l'Autriche (nivôse an VIII) ou à l'occasion du traité d'El Arisch (pluviôse an VIII), la presse officielle rappelait les diverses atteintes portées aux députés ou aux diplomates de la République par l'Autriche; les mêmes exemples revenaient sans cesse: on dénonçait l'arrestation du ministre Beurnonville et des représentants Bancal, Quinette, Camus et Lamarque par Dumouriez, et leur incarcération en Autriche (avril 1793); on évoquait l'arrestation, sur ordre du gouvernement de Milan et en territoire neutre, des plénipotentiaires Sémonville et 
Maret en route pour négocier avec Venise, Florence et Naples (juillet 1793); on rappelait la destruction du drapeau français et les menaces faites par les Viennois à l'ambassadeur Bernadotte après Campoformio (avril 1798), et bien sûr l'assassinat de Bonnier et Roberjot à Rastadt ${ }^{28}$. Ces rappels permettaient de souligner la « perfidie » du «machiavélique cabinet de Vienne » ${ }^{29}$, dans laquelle certains, comme Merlin de Douai, voulaient voir une tradition nationale, déjà perceptible au temps de Charles Quint, lorsque les ambassadeurs français César Frégose et Antoine Rincon avaient été assassinés par ordre du gouverneur du Milanais ${ }^{30}$.

10 À la « perversité », c'est la « loyauté » républicaine que l'on opposait ; jamais, assuraiton au lendemain de l'assassinat de Rastadt, la France révolutionnaire n'avait violé les principes du droit des gens ${ }^{31}$; d'aucuns, évoquant l'exemple de Cellamare, soutenaient même que la Régence, et de manière générale la monarchie du dernier siècle, les avaient également respectés ${ }^{32}$. Ponctuellement, cette partie de l'idéal républicain, cette soumission revendiquée au droit des nations, devenait une tradition française et permettait de faire communier l'ancien et le nouveau régime dans de mêmes principes. Certes, notamment au lendemain de la journée du 30 prairial an VII (18 juin 1799), dans les dénonciations admises contre les directeurs démissionnaires, l'on reprocha au gouvernement d'avoir transgressé ces règles; dans la dénonciation signée Deleschaux, dont l'esprit mesuré recherchait l'union des républicains ${ }^{33}$, l'on reprochait aux directeurs d'avoir attaqué l'empire ottoman et la Suisse sans respecter les formes prescrites par les usages (manifeste ou déclaration préalable), et sans motifs permettant de justifier le déclenchement d'un conflit ${ }^{34}$. La critique entraîna cependant, chez Merlin, Reubell et La Révellière-Lépeaux une réponse organisée dans laquelle les ex-directeurs, implicitement, ou avec force détails (La Révellière), disaient leur attachement aux formes et aux règles du droit des nations ${ }^{35}$. En l'été 1799 , chez les néojacobins comme chez les «triumvirs menacés d'une mise en accusation, c'était le même attachement proclamé au droit des gens qui était exprimé.

11 Au lendemain du 18 Brumaire, cette conviction que la France se devait, par esprit républicain mais aussi par tradition nationale, de respecter le droit des gens n'avait pas disparu ; au contraire, elle paraissait même se renforcer. À cet égard, l'offre de paix directement adressée par Bonaparte au roi d'Angleterre, le 5 nivôse an VIII (25 décembre 1799), était parfois perçue comme un rejet d'une diplomatie secrète et trompeuse, déjà formulé par l'Assemblée constituante; sans évoquer pourtant la reconnaissance des traités secrets en l'an III ${ }^{36}$, l'auteur de l'Examen impartial des deux lettres $d u$ premier consul [...] et $d u$ roi d'Angleterre sur la paix se réjouissait que le gouvernement se dirigeât "vers une politique élevée et morale, vers une diplomatie juste et généreuse, franche et digne des beaux jours de l'Europe " ${ }^{37}$. La République, et peut-être la France - celle d'avant et d'après 1792 -, se voulait fidèle à un droit des gens épuré, ennobli... De part et d'autre du 18 Brumaire, ce n'était pas la proclamation des principes qui changeait; tout au plus pouvait-on percevoir une altération des cris de vengeance en cas de violation extérieure du droit des nations.

12 En l'an VII, en effet, au lendemain de l'assassinat de Bonnier et de Roberjot, la République se présentait comme la gardienne et la vengeresse du droit des gens. En prairial an VII, lors de la fête organisée en l'honneur des plénipotentiaires de Rastadt, Merlin de Douai avait dit :

«Il n'est point sur la terre d'institutions protectrices des droits des peuples, et qui puissent atteindre les crimes des gouvernements : mais si la justice universelle peut trouver un digne organe dans une nation devenue l'asile de la foi et de l'honneur, 
cette enceinte même n'est-elle pas un tribunal auguste où doit être publié devant l'univers, et sous les auspices du juge suprême, le jugement irrévocable des peuples et de la postérité ? $\|^{38}$

13 L'indignation de la République ne se voulait pas seulement celle d'une nation " outragée », mais celle d'un pays indigné au nom de l'humanité entière ; l'affaire ne concernait pas uniquement la France, mais l'ensemble des nations. C'est au nom de la République, c'est également au nom de l'humanité que le directoire exécutif ou certains membres des Conseils demandaient la «vengeance » du crime autrichien ${ }^{39}$.

14 À la suite de l'affaire de Rastadt, la réaction de la République se voulait d'une grande fermeté, et se justifiait par la portée universelle et l'importance fondatrice du droit des nations ${ }^{40}$ : aux Cinq-Cents, Sherlock avait proposé la constitution d'un tribunal international chargé de poursuivre «les crimes commis contre le droit des gens; la proposition, déjà envisagée par des auteurs du XVIII ${ }^{\mathrm{e}}$ siècle, avait été accueillie par les cris : « des bayonnettes !... des bayonnettes !... des coups de canon !... " ${ }^{41}$. Quelques jours plus tard, Le Moniteur annonçait que les troupes de la rive gauche du Rhin avaient décidé de ne " plus donner de quartier » et de ne plus faire de prisonniers ; "tous les ennemis qu'on a pu atteindre [poursuivait l'article], ont été hachés " ${ }^{42}$. Comment ne pas rapprocher cette décision et ses justifications théoriques des débats des Jacobins et de la Convention sur les crimes des Anglais ${ }^{43}$; le 7 prairial an II (26 mai 1794), pour châtier une nation qui, par l'acceptation tacite des violations du droit de son gouvernement, s'était exclue de l'humanité, on avait décidé de ne plus faire "aucun prisonnier anglais ou hanovrien ", c'est-à-dire de ne laisser la vie sauve à aucun de ces irréductibles ennemis de la République et du monde! Certes, cinq ans plus tard, la décision de procéder à une guerre à outrance contre certains ennemis de "l'humanité » avait été prise par les états-majors militaires, et non par les représentants de la nation, et la nature de la décision avait probablement évolué ${ }^{44}$; elle procédait pourtant, en partie tout au moins, d'un même raisonnement, d'une même conception d'une société des nations cimentée par des règles communes auxquelles la France se conformait, et qu'elle se devait de faire respecter.

Évidemment, l'importance de l'événement, de même que les difficultés militaires et l'instabilité politique intérieure, expliquent la vigueur des propos et des menaces; ainsi, après Brumaire, les discours sur l'affaire Blackwell paraissent trahir un apaisement des réactions françaises. En février 1800, le prochain procès de Barthélémy Blackwell entretenait ponctuellement le débat sur le droit des nations. Cet Irlandais naturalisé français, qui avait été arrêté à Hambourg pour ses activités politiques au sein des Irlandais unis, avait subi un emprisonnement d'une année avant d'être livré aux Anglais qui entendaient le juger pour trahison ${ }^{45}$. Dans cette arrestation d'un citoyen français en territoire étranger, la République voyait une violation caractérisée du droit des gens ; cependant, l'indignation publique, toujours présente, s'exprimait désormais en des termes mesurés. Dans le précis pour Barthélémy Blackwell publié par Le Moniteur, le 23 pluviôse an VIII (12 février 1800), on appelait certes la « vengeance du gouvernement français » contre Hambourg, mais l'auteur se contentait d'espérer que le gouvernement anglais reviendrait bientôt "à la raison" ${ }^{46}$; parce que le crime ne frappait pas comme celui de Rastadt, mais aussi parce que la situation politique du pays changeait, les réactions françaises s'apaisaient, sans pour autant que l'on abandonne le discours sur le nécessaire respect du droit des gens. En l'an VII comme en l'an VIII, il apparaissait comme un élément central de l'identité républicaine, au même titre que le rejet des guerres de conquête. 
La « modération » contre « l'ambition »

16 La guerre de la seconde coalition contre la France était dépeinte comme celle de l'ambition contre le désintéressement; pour dénier toute légitimité aux objectifs des coalisés, leurs buts de guerre étaient présentés comme juridiquement et moralement injustifiables. Cette dénonciation rappelait le rejet des guerres entreprises dans l'intention de faire des conquêtes par l'Assemblée constituante. Le discours, non dénué de contradictions, ne faisait pourtant aucune allusion aux premiers temps de la Révolution, ni d'ailleurs à l'époque conventionnelle, bien qu'il parût se nourrir avant tout des théories élaborées dans les débats de l'an III, notamment lors des discussions qui avaient précédé la réunion de la «Belgique à la France, le 9 vendémiaire (1er octobre 1795) ${ }^{47}$.

Une fois encore, l'image des principes républicains se construisait par le rejet de pratiques réprouvées; implicitement, de part et d'autre du 18 Brumaire, la guerre de conquête était rejetée comme injuste. L'un des premiers reproches adressés aux coalisés était ainsi leur « ambition » qualifiée de "dévorante " ${ }^{48}$ ou de "colossale " ${ }^{49}$, dans laquelle on isolait surtout des projets d'extension territoriale. Au début de l'année 1800 étaient dénoncées les vues de la Russie sur les îles vénitiennes, les conquêtes indiennes et la domination maritime des Anglais, la volonté présumée de l'Autriche de contrôler l'Italie, mais aussi la Suisse et l'Allemagne ${ }^{50}$. Ces desseins paraissaient enlever toute légitimité à la coalition, et notamment au rapprochement austro-russe que l'on présentait comme une «association impie des monarques [...] formée par l'ambition, cimentée par le crime [de Rastadt] " ${ }^{51}$. Le rejet de la guerre de conquête, on s'en rend compte, n'était guère explicite; à aucun moment les écrits des philosophes ou des jusnaturalistes, les débats ou les engagements solennels de l'Assemblée constituante n'étaient rappelés pour rejeter les objectifs attribués aux coalisés ; à aucun moment, un principe, clairement formulé, n'était opposé aux pratiques ennemies. À « [l']ambition démasquée " des coalisés on opposait pourtant la "modération soutenue " de la République $^{52}$; l'imprécision des termes employés rappelait que l'extension récente du territoire français n'avait pu se faire sans invoquer des règles nouvelles qui, en certaines occasions, acceptaient comme juste la réunion de territoires sans le consentement immédiat des populations concernées.

18 L'attachement peu explicite au rejet des guerres de conquête cachait en fait une vision complexe et ambivalente des buts de guerre, en grande partie héritée des débats de l'an III. Le trait le plus marquant de cet héritage est le rappel, notamment en floréal an VII, de l'existence des «droits du peuple vainqueur » que les thermidoriens avaient définis comme la possibilité laissée au peuple qui avait enduré une guerre injuste de réclamer une compensation raisonnable, éventuellement territoriale, à titre de dédommagement du tort subi ; en l'an III, et probablement encore cinq ans plus tard, l'on pensait que ce nouveau droit ne contrariait pas le rejet de la guerre entreprise dans le but de faire des conquêtes, car la réunion du Limbourg et du Luxembourg à la République, par exemple, ne paraissait pouvoir être comparée avec le dernier partage de la Pologne ${ }^{53}$. À la différence de ce qui s'était passé à l'automne 1795, le rappel de ces droits n'était cependant pas destiné à justifier des réunions, dont on ne parlait plus, mais à souligner que la République, par souci de «modération », ne faisait pas toujours un plein usage des droits que ses victoires lui laissaient, à la manière de Dubois-Dubais qui, le 16 floréal an VII (5 mai 1799), pouvait dire: «Vous avez respecté des rois perfides sur lesquels vous pouviez exercer les droits d'un vainqueur justement irrité [...]» ${ }^{54}$. Le 
désintéressement d'une France ne rejetant pas nécessairement les compensations, et justifiant ainsi une partie de ses agrandissements passés, s'opposait à «l'ambition » de ses ennemis; la République prétendait combattre dans l'intérêt de la liberté et de la civilisation.

République, liberté et civilisation

19 La guerre menée par la République était présentée comme purement défensive, et une fois encore l'intérêt des Français paraissait rejoindre celui de l'humanité. Bien sûr, face aux armées coalisées, on présentait d'abord les soldats de la République comme les défenseurs de ses valeurs fondamentales, la liberté et la propriété, mais aussi de son indépendance et de son honneur ${ }^{55}$; comme l'écrivait, au début de l'année 1800, l'auteur de L'examen impartial des deux lettres du premier consul [...] et du roi d'Angleterre sur la paix, la France «fai[sai]t la guerre défensive de son indépendance et de ses droits " ${ }^{56}$. Dans ce combat, dont la nature était présentée comme en partie idéologique, la cause de la République était pourtant assimilée à celle de l'humanité entière : en voulant briser la République, écrivait Merlin, les coalisés espéraient «étouffer sur toute la surface du globe la liberté et les lumières " ${ }^{57}$; après Brumaire, ce discours ne disparaît aucunement et les coalisés continuent à être présentés comme les ennemis naturels de la liberté ${ }^{58}$. La lutte des Français pour éviter le retour de «l'esclavage » ${ }^{59}$ se doublait d'un enjeu qui intéressait l'ensemble du « monde».

La France, en effet, ne se contentait pas de défendre sa liberté, elle prétendait également l'offrir aux peuples amis et participer à la diffusion inéluctable de ce principe dont on plaçait les premiers progrès au temps de la Révolution américaine ${ }^{60}$; à la manière des Insurgents, on continuait à considérer que l'indépendance des 13 colonies anglaises avait ouvert un nouvel âge dans l'histoire de l'humanité ${ }^{61}$. De part et d'autre du 18 Brumaire, les Républicains se présentaient comme les principaux apôtres de la liberté. Au printemps 1800, les campagnes et les victoires militaires en Italie entretenaient ce discours : le 17 prairial (6 juin 1800), Bonaparte haranguait ses troupes en leur demandant de rendre « la liberté et l'indépendance au peuple de Gênes » ${ }^{62}$; le 9 messidor (28 juin 1800), le général Dupont installait le gouvernement provisoire du Piémont en saluant le retour de « la liberté, [de] la sécurité et [du] bonheur » dans ce territoire ${ }^{63} \ldots$ La liberté, inévitablement portée par les armées de la République, paraissait appelée à triompher dans " toutes les parties du monde " ${ }^{64}$, en même temps que la «civilisation » que défendait la France face à la Russie ou à l'Empire ottoman, dont on dénonçait la barbarie ${ }^{65}$ et l'ignorance ${ }^{66}$.

Le discours libérateur et civilisateur des Français n'était cependant plus nécessairement un chant martial; l'on convenait certes que la guerre facilitait la diffusion de la liberté, car on assurait que les peuples étaient prêts à l'accueillir ${ }^{67}$, mais les ministres ou la presse officielle se défendaient, en l'an VII comme en l'an VIII, de vouloir perpétuer une guerre aux rois. Ces projets, jugés démesurés et d'autant plus dangereux qu'on souhaitait une paix prompte et victorieuse, étaient rejetés sur la Convention ou sur les Jacobins ; ainsi, Talleyrand dénonçait ces hommes :

«qui étaient impatiens de mouvements révolutionnaires dans toutes les parties du monde, qui adressaient inconsidérément à toutes les puissances les injures les plus absurdes et les plus impolitiques, qui ne semblaient s'occuper qu'à entraver toute négociation, qui se plaisaient à répandre sans cesse dans les feuilles publiques, cette assertion si funeste au repos de l'Europe: que les républiques et les rois sont essentiellement en guerre $[. .]. »{ }^{68}$ 
Ce discours, tenu dès l'an VII, était généralement bien accueilli, et les étrangers reconnaissaient parfois à la République l'abandon de pratiques et d'objectifs jugés dangereux pour l'équilibre européen. Dans son Coup d'œil politique sur le continent (1800), le Genevois Saladin applaudissait à l'abandon " du système de républicanisme général » ${ }^{69}$, tandis qu'en janvier 1800, le Morning chronicle savait gré aux consuls de ne pas chercher à insulter ou à "avilir » les gouvernements avec lesquels la France était en guerre ${ }^{70}$. Par sa diplomatie et ses discours, la République, tout en continuant à se proclamer libératrice et civilisatrice, entendait se doter d'une image acceptable aux yeux de ses voisins.

image, cependant, se transformait progressivement au tournant du siècle. Bonaparte, déjà héroïsé, paraissait devenir le défenseur privilégié de la République et de ses valeurs ${ }^{71}$; en Égypte, pour reprendre des propos du ministre de la Guerre Berthier, c'était «Bonaparte» qui affranchissait par la victoire «la terre natale des arts » et y apportait " entouré de savants et de guerriers, les lumières et la civilisation » ${ }^{72}$... Ce processus d'incarnation des principes républicains dans un homme n'en était pourtant qu'à ses débuts, et la pacification intérieure et extérieure devait un temps l'accentuer sans pour autant jamais l'achever; dans les félicitations adressées aux consuls au lendemain de la paix d'Amiens, les attributs de la République paraissaient ainsi ponctuellement devenir ceux de Bonaparte, "protecteur des sciences et des arts » ${ }^{73}$, « pacificateur du monde " ${ }^{74}$, «bienfaiteur de l'humanité » ${ }^{75}$ ou encore " père des peuples ${ }^{76}$

Respecter la souveraineté des peuples

Lorsque les auteurs de l'an VII et de l'an VIII évoquaient la liberté apportée par la République aux nations voisines, ils rappelaient son engagement à respecter l'indépendance des peuples amis, c'est-à-dire leur liberté politique interne. Ce principe, bien que malmené dès les premières années de la Révolution, continuait à être proclamé et ne pouvait être renié tant il était lié à l'idée de souveraineté nationale ; comme à l'époque constituante, il paraissait difficile d'isoler le droit public national et international ${ }^{77}$.

Le respect nécessaire de la souveraineté des peuples, évidemment hérité de la pensée du XviII ${ }^{e}$ siècle, avait été proclamé lors des débats de l'Assemblée constituante sur la Corse (janvier 1790), sur les princes possessionnés d'Alsace (octobre 1790) et sur la réunion d'Avignon et du Comtat Venaissin à la France; mêlé au rejet de la guerre de conquête, cette reconnaissance de la souveraineté des peuples était apparue comme une véritable révolution, que les juristes et les historiens ont sacralisée en y voyant l'origine du principe du «droit des peuples à disposer d'eux-mêmes " ${ }^{78}$. L'expression, un temps validée par l'usage, n'est plus unanimement reconnue tant il est vrai que sa formulation remonte essentiellement aux 14 points du président Wilson, et qu'elle ne s'est inscrite dans le droit international qu'avec les chartes de l'Atlantique (août 1941) et des Nations Unies (juin 1945) ${ }^{79}$; son remplacement par la notion de "droit des peuples ", dont l'usage se retrouve dans les textes, n'enlève malheureusement pas tout risque de confusion puisque depuis les années 1960, l'élargissement des droits reconnus aux peuples - droits sociaux, économiques, culturels... - a conduit certains juristes à substituer à l'expression "droit des peuples à disposer d'eux-mêmes ", jugée trop étroite, la notion de "droit des peuples ${ }^{80}$. Quelle que soit l'expression employée, il suffit, pour éviter tout malentendu, de préciser que le droit de souveraineté reconnu aux peuples par les constituants, et toujours d'actualité de part et d'autre de Brumaire,

Annales historiques de la Révolution française, 318 | octobre-décembre 1999 
avait deux dimensions ; la première, d'ordre interne, reconnaissait aux peuples le libre choix de leur type de gouvernement et de leurs responsables politiques; la seconde, que l'on qualifierait aujourd'hui de droit public international, leur permettait, s'ils pouvaient apparaître comme politiquement constitués, de demander leur réunion à un État voisin. Aucune reconnaissance d'un éventuel droit de sécession n'était envisagée, ni en 1790, ni dans les années suivantes.

$\mathrm{Au}$ début du Consulat, fière de son héritage, la République se présentait comme la mère de ce nouveau « droit des peuples»:

«Ce peuple, disait Petiet en s'adressant à la Consulta de la République cisalpine, en messidor an VIII, a révélé au monde ce grand principe de toutes les lois humaines, que la volonté générale est la seule autorité légitime ; non content de la proclamer, il le professe, et après avoir affranchi votre nation d'un joug étranger, il s'en remet à elle du choix de son organisation politique. ${ }^{81}$

La France l'avait révélé au monde, et elle le respectait... C'est ce discours officiel que les généraux de la République pouvaient répéter en Italie du Nord, au printemps de l'année 1800, à Milan, à Gênes et à Turin où, en messidor an VIII, le général Dupont affirmait : "La République française respecte et protège les droits de la nation piémontaise ${ }^{82}$ ». L'indépendance et la souveraineté des peuples « libérés » étaient officiellement mises sous la sauvegarde de la République.

Ce discours, sans cesse répété, ne se reflétait bien évidemment pas nécessairement dans la pratique des gouvernements, pas plus en l'an VIII qu'en l'an VII ${ }^{83}$; son importance était cependant telle qu'aucun homme public ne pouvait le rejeter. À cet égard, les débats qui avaient suivi la journée du 30 prairial an VII (18 juin 1799) sont particulièrement révélateurs. Certaines dénonciations des "triumvirs", reprises en partie par Montpellier de l'Aude, faisaient explicitement référence aux violations de la souveraineté des peuples de Suisse, d'Italie et de Batavie; elles dénonçaient, à la manière de Ruelle, les «perfidies » de la diplomatie directoriale ${ }^{84}$, jugées indignes de la République et justifiant une mise en accusation des directeurs déchus; plus explicitement, Deleschaux présentait comme deuxième chef d'accusation la violation de «la souveraineté des peuples» et en donnait pour preuve les modifications arbitrairement apportées aux «constitutions» batave et cisalpine, ainsi que la violation de l'indépendance du peuple romain ${ }^{85}$. C'étaient les interventions dans la politique intérieure de ces États qui étaient dénoncées, et notamment les coups d'État menés dans les républiques sœurs, qu'ils le fussent par des décisions autonomes d'agents du pouvoir ou qu'ils aient été commandés de Paris ${ }^{86}$.

Ces attaques contre les directeurs n'exprimaient pas seulement les convictions des néojacobins ; elles permettaient aussi aux «triumvirs » de rappeler leur attachement à ce qu'ils considéraient comme des principes fondateurs de la République. Dans leurs réponses, Merlin, La Révellière et Reubell s'attardaient sur leur souci de respecter l'indépendance des républiques amies : ils assuraient qu'ils avaient simplement usé de persuasion pour obtenir la modification de la constitution batave ${ }^{87}$; que dans la République Cisalpine, la prétendue " constitution » n'était qu'une ordonnance militaire sans légitimité populaire, et que les autorités provisoires ne pouvaient y être considérées comme un gouvernement représentant le vœu du souverain ${ }^{88}$; que la « constitution romaine » n'avait pas plus de légitimité politique que celle de la Cisalpine ${ }^{89}$. Outre la volonté des anciens directeurs d'échapper à une mise en accusation, ces 
propos trahissaient leur volonté de proclamer un attachement à des principes jugés fondamentaux.

L'étude de la politique extérieure de la République, dans les dernières années de la Révolution, peut prendre plusieurs chemins qui tous ont leur légitimité ; l'observation des faits, l'approche philosophique, l'examen de la perception de la politique menée en territoire étranger et de l'image que les Français se faisaient des autres peuples ${ }^{90}$, ou l'analyse des représentations de l'idéal républicain concourent à sa connaissance et à sa compréhension, qui passe par la prise en compte conjointe de ces approches. Dans le domaine des relations entre les peuples, l'idéal républicain que diffusaient la presse ou les responsables politiques n'est pas à négliger, bien qu'il puisse paraître éloigné des pratiques ${ }^{91}$; de même, l'existence, en Angleterre, de discours concurrents sur le nécessaire respect du droit des gens ou de la souveraineté des nations ${ }^{92}$, ne doit pas faire oublier que les républicains français concevaient leurs principes comme profondément neufs et originaux.

31 L'analyse de l'idéal républicain permet ainsi de rappeler que, de part et d'autre de Brumaire, même si les évolutions liées à la montée d'un homme jugé providentiel ne doivent pas être sous-estimées, la République voulait se définir par le respect proclamé de certaines valeurs. Plusieurs traits caractérisent cet idéal : bien que les références au droit naturel subsistent, notamment dans l'analyse des sources du droit des gens, les allusions à la fraternité entre les peuples ou à la République universelle ont disparu ; parallèlement, la République, tout en demeurant implicitement fidèle aux principes affichés à l'époque constituante, ose parfois revendiquer l'héritage d'une France d'Ancien Régime jugée loyale et respectueuse du droit des nations. Dans les discours, la République n'est pourtant pas détachée de ses origines révolutionnaires, bien au contraire; on continue de la montrer favorable à la propagation de la liberté et respectueuse de la souveraineté des peuples récemment "affranchis». L'acceptation des «droits du peuple vainqueur », qui ne voulait pas s'assimiler à une reconnaissance d'un droit de conquête, rattachait pourtant clairement cet idéal à l'an III. En 1800 encore - mais l'idée fut-elle un jour totalement abandonnée? ${ }^{93}$-, la France se considérait comme la patrie des Droits de l'homme, comme la gardienne du droit des nations et comme le défenseur naturel du droit des peuples.

\section{NOTES}

1.Maurice AGULHON, Marianne au combat. L'imagerie et la symbolique républicaines de 1789 à 1880, Paris, Flammarion, 1979, pp. 33-34 et 46-49.

2.Discours de Garat au Conseil des Anciens, le 16 floréal an VII (5 mai 1799). Le Moniteur, $\mathrm{n}^{\circ} 231$, du 21 floréal, p. 941.

3. Claude NICOLET, L'idée républicaine en France. Essai d'histoire critique, Paris, Gallimard, 1982, p. 30 ; voir aussi, sur l'idée républicaine, pp. 9-43. 
4.Sur les liens entre image et idéal : Maurice AGULHON, « Marianne, objet de culture ", dans Jean-Pierre RiouX et Jean-François sIRINELLI, Pour une histoire culturelle, Paris, Seuil, 1997, not. p. 122.

5.T.C.W. BLANNING, The French Revolutionnary Wars. 1787-1802, London, Arnold, 1996, pp. 47-51.

6.Acception définie par Serge BERSTEIN, « La culture politique », dans Jean-Pierre RIOUX et Jean-François SIRINELLI, op. cit., pp. 371-386.

7.Voir : Pierre-Yves BEAUREPAIRE, La République universelle des francs-maçons. De Newton à Metternich, Rennes, éd. Ouest-France, 1999 ; Armand MATTELART, Histoire de l'utopie planétaire. De la cité prophétique à la société globale, Paris, La découverte, 1999 ; Marc BELISSA, Fraternité universelle et intérêt national (1713-1795). Les cosmopolitiques du droit des gens, Paris, Kimé, 1998 ; Sophie WAHNICH, L'impossible citoyen. L'étranger dans le discours de la Révolution française, Paris, Albin Michel, 1997.

8.Sur l'usage de ces deux expressions, voir les débats sur l'assassinat des plénipotentiaires français à Rastadt : notamment Le Moniteur, $\mathrm{n}^{\circ} 227,17$ floréal an VII, p. 925 ; id., n 228, 18 floréal, p. 928 ; id., n²29, 19 floréal, pp. 933-934 ; id., n² 230, 20 floréal, p. 937.

9.Bailleul au Conseil des Cinq-Cents le 16 floréal an VII (5 mai 1799). Le Moniteur, $n^{\circ} 229$, du 19 floréal, p. 934.

10.P.V. Benoist, Sur l'exécution du traité fait par le général Kléber avec le grand vizir. Le Moniteur, $\mathrm{n}^{\circ}$ 212, 2 floréal an VIII, p. 859.

11.Discussion de l'expression : « La Révolution est fixée aux principes qui l'ont commencée ; elle est finie »; Le Moniteur, $\mathrm{n}^{\circ}$ 115, du 25 nivôse an VIII, p. 456. 12.Sur le droit des gens du XVIII ${ }^{e}$ siècle, voir Marc BELISSA, Fraternité universelle..., op. cit., not. pp. 20-119; Jean-Pierre BoIs, L'Europe à l'époque moderne, Paris, A. Colin, pp. 221-234. Pour une approche juridique : Louis CAVARÉ, Le droit international public positif, Paris, Pedone, 3e éd., 1966, t. 1, not. pp. 48-62. Pour une approche des œuvres, voir le toujours utile A. PILLET et alii, Les fondateurs du droit international, Paris, V. Giard et E. Brière, 1904. 13.Lettre de François de Neufchâteau à Jean Debry, du 17 floréal an VII (6 mai 1799), dans P. MONTARLOT et L. PINGAUD, Le congrès de Rastadt (11 juin 1798-28 avril 1799).

Correspondance et documents, Paris, A. Picard et fils, t. 3, 1913, p. 234. Message du directoire exécutif aux Conseils des Anciens et des Cinq-Cents, du 16 floréal an VII (5 mai 1799) ; repoduit dans Le Moniteur, $n^{\circ} 228$, du 18 floréal, p. 928.

14.P.V. Benoist, op. cit. ; reproduit dans Le Moniteur, $n^{\circ} 212$, du 2 floréal an VIII, p. 859. 15.Sur les visions différentes des relations entre Révolution et droit naturel, voir Florence GAUTHIER, Triomphe et mort du droit naturel en Révolution. 1789-1795-1802, Paris, PUF, 1992, not. pp. 143-145 et Claude NICOLET, op. cit. ; pp. 333-342 et 344-346.

16.Message du directoire exécutif aux Conseils des Anciens et des Cinq-Cents du 16 floréal an VII (5 mai 1799). Le Moniteur, $\mathrm{n}^{\circ}$ 228, du 18 floréal, p. 928.

17.Sur G.F. Martens et son Précis du droit des gens moderne de l'Europe (1788), voir Louis CAVARÉ, op. cit., t. 1, pp. 61-62 ; A. PILLET et alii, op. cit., not. pp. 611-613.

18.Détails transmis par Jean Debry sur l'affaire de Rastadt. Le Moniteur, $n^{\circ} 238$, du 28 floréal an VII, p. 969.

19.Heurtault-Lamerville au Conseil des Cinq-Cents, le 20 prairial an VII (9 mai 1799). Le Moniteur, $\mathrm{n}^{\circ}$ 262, 22 prairial, p. 1068.

20.Id., p. 1070 ; voir aussi l'intervention de Bailleul aux Cinq-Cents le 16 floréal an VII (5 mai 1799) : Le Moniteur, $n^{\circ} 229$, du 19 floréal, p. 933. 
21.Par exemple : Le Moniteur, $\mathrm{n}^{\circ}$ 228, 18 floréal an VII, p. 928 ; id., $\mathrm{n}^{\circ} 229,19$ floréal, $\mathrm{p}$. 933 ; id., n² 234, 24 floréal, p. 952.

22.Bailleul aux Cinq-Cents, le 16 floréal an VII (5 mai 1799), pour la citation ; Le Moniteur, $n^{\circ} 229,19$ floréal, p. 933. Voir aussi le discours de Gourdan aux Anciens, le 20 prairial an VII (8 juin 1799); id., n² 263, 23 prairial, p. 1073.

23.Heurtault-Lamerville au Conseil des Cinq-Cents, le 20 prairial an VII (8 juin 1799). Le Moniteur, $\mathrm{n}^{\circ}$ 262, 22 prairial, p. 1068.

24.Bailleul aux Cinq-Cents, le 16 floréal an VII (5 mai 1799), pour la citation; Le Moniteur, $\mathrm{n}^{\circ} 229,19$ floréal, p. 934.

25. « Monstres » : l'administration du département de la Seine à ses concitoyens, s.d. (Le Moniteur, $\mathrm{n}^{\circ}$ 234, 24 floréal an VII, p. 952) ; « cannibales » et « sauvages » : HeurtaultLamerville au Conseil des Cinq-Cents, le 20 prairial -8 juin 1799- (id., $\mathrm{n}^{\circ} 262,22$ prairial, p. 1068) ; «barbares » : Gourdan au Conseil des Anciens, le 20 prairial an VII -8 juin 1799-(id., n² 263, 23 prairial, p. 1073).

26.Gourdan au Conseil des Anciens, le 20 prairial an VII (8 juin 1799). Le Moniteur, $\mathrm{n}^{\circ}$ 264, du 24 prairial an VII, p. 1077.

27.Discours prononcé par Merlin de Douai au Champ-de-Mars, le 20 prairial an VII (8 juin 1799). Le Moniteur, $\mathrm{n}^{\circ}$ 265, du 25 prairial, réimpression, t. 29, p. 707.

28.Sur ces rappels, voir : sur l'assassinat de nos plénipotentiaires à Rastadt, Le Moniteur, $\mathrm{n}^{\circ} 227,17$ floréal an VII, p. 925 ; Examen impartial des deux lettres du premier consul de la République française et du roi d'Angleterre sur la paix, id., $n^{\circ} 124$, du 4 pluviôse an VIII, p. 493 ; sur l'exécution du traité fait par le général Kléber avec le grand vizir, id., $\mathrm{n}^{\circ}$ 212, 2 floréal an VIII, p. 860.

29.Proclamation du Directoire exécutif sur l'assassinat de Rastadt (Le Moniteur, $\mathrm{n}^{\circ} 230$, du 20 floréal an VII, p. 937) ; Dubois-Dubais au Conseil des Anciens, le 16 floréal an VII 5 mai 1799 - (id., p. 938) ; Heurtault-Lamerville aux Cinq-Cents, le 20 prairial an VII (8 juin 1799) (id., $\mathrm{n}^{\circ} 262$, du 22 prairial, p. 1069).

30.Charles Quint, en 1540, aurait donné l'ordre d'intercepter les deux représentants de François Ier. Lettre de Merlin de Douai à Jean Debry, du 15 floréal an VII (4 mai 1799), dans P. MONTARLOT et L. PINGAUD, op. cit., t. 3, p. 229.

31.Adresse du directoire exécutif à tous les peuples et à tous les gouvernements, du 18 floréal an VII - 7 mai 1799 - (Le Moniteur, n² 230, du 20 floréal, p. 937).

32.Sur l'assassinat de nos plénipotentiaires. Le Moniteur, n 227, 17 floréal an VII, p. 925. 33. Sur cette dénonciation : Bernard GAINOT, Le mouvement néo-jacobin à la fin du Directoire. Structure et pratiques politiques, Thèse, Paris I, 1993, dactylographiée, p. 108.

34.Dénonciation de Deleschaux, du 25 messidor an VII (13 juillet 1799). Le Moniteur, $\mathrm{n}^{\circ}$ 324, du 24 thermidor, p. 1318.

35.Réponses de L. M. Révellière-Lépeaux, aux dénonciations portées au corps législatif contre lui et ses anciens collègues. 15 thermidor an 7, imp. H.J. Jansen, s.d., not. pp. 7-8 (Bibl. nat. de France, $8^{\circ}$ LB42 742). Voir aussi : Ph. Ant. Merlin, membre de l'Institut national, au Conseil des Cinq-Cents, Paris, Desenne, an VII, 45 pp. (Bibl. nat., $8^{\circ}$ LB42 $2324)$; et la justification de Reubell dans Bernard Nabonne, La diplomatie du Directoire et Bonaparte d'après les papiers inédits de Reubell, Paris, La nouvelle édition, 1951, not. pp. 163-180 (Jean-René Suratteau et Alain Bischoff, Jean-François Reubell. L'alsacien de la Révolution française, s.l., Editions du Rhin, 1995, pp. 376-382).

36.Décret de la Convention du 27 ventôse an III (17 mars 1795).

37.Examen impartial des deux lettres..., op. cit. ; compte rendu dans Le Moniteur, $\mathrm{n}^{\circ} 124$, du 4 pluviôse an VIII, pp. 493-494. 
38. Merlin de Douai, le 20 prairial an VII (8 juin 1799). Le Moniteur, $\mathrm{n}^{\circ}$ 265, du 25 prairial an VII, réimpression, t. 29, p. 707.

39.Par exemple : message du Directoire exécutif aux Conseils des Anciens et des CinqCents, du 16 floréal an VII - 5 mai 1799 - (Le Moniteur, n²28, du 18 floréal, p. 928) ; débats au Conseil des Cinq-Cents le 16 floréal (id., $\mathrm{n}^{\circ}$ 229, du 19 floréal, p. 933).

40.Cf. supra.

41.Séance du Conseil des Cinq-Cents du 16 floréal an VII (5 mai 1799). Le Moniteur, $\mathrm{n}^{\circ}$ 229, du 19 floréal, p. 933.

42.Le Moniteur, $\mathrm{n}^{\circ}$ 232, du 22 floréal an VII, p. 943.

43.Sophie WAHNICH, op. cit., pp. 237-241; Marc BELISSA et Sophie WAHNICH, « Les crimes des Anglais : trahir le droit ", Annales historiques de la Révolution française, $\mathrm{n}^{\circ}$ 300, 1995, pp. 233-248.

44.Le rejet d'un peuple-tyran n'apparaît évidemment pas ici ; voir Marc BELISSA et Sophie WAHNICH, op. cit., not. pp. 240-248.

45.Sur Blackwell : A.V. ARNAULT, A. JAY, E. JOUY et J. NORVINS, Biographie nouvelle des contemporains, Paris, Bibliothèque historique, t. 3, 1821, p. 44.

46. Le Moniteur, $\mathrm{n}^{\circ}$ 143, du 23 pluviôse an VIII, p. 569.

47.Sur ces débats : Hervé LEUWERS, «Droit des peuples et droits du peuple vainqueur. Caractères et justifications de la seconde réunion de la Belgique à la France (1795) », dans Justice et institutions françaises en Belgique (1795-1815), Hellemmes, Ester, 1996, pp. 203-218 ; Marc BELISSA, Fraternité universelle..., op. cit., pp. 426-433.

48.Par Dubois-Dubais, à propos de l'Angleterre et de l'Autriche, au Conseil des Anciens, le 16 floréal an VII - 5 mai 1799 - (Le Moniteur, $\mathrm{n}^{\circ}$ 230, du 20 floréal, p. 938).

49.Examen impartial des deux lettres..., op. cit. (Le Moniteur, $\mathrm{n}^{\circ} 124$, du 4 pluviôse an VIII, p. 494).

50.Coup d'œil politique sur l'Europe (attribué à J. Blanc de Volx); compte rendu dans Le Moniteur, n 235, du 25 floréal an VIII, p. 950 ; voir aussi id., n 124, du 4 pluviôse an VIII, p. 494 ; id., article du $n^{\circ} 211$, du 1er floréal an VIII, p. 855.

51.Proclamation du directoire exécutif sur l'assassinat de Rastadt, datée du 17 floréal an VII (6 mai 1799); publiée dans Le Moniteur, n 230, du 20 floréal an VII, p. 936.

52.Adresse du Directoire exécutif à tous les peuples et à tous les gouvernements; Le Moniteur, $\mathrm{n}^{\circ}$ 230, du 20 floréal an VII, p. 937.

53.Sur la définition des droits du peuple vainqueur par Portiez de l'Oise, Merlin de Douai, Roberjot ou Eschassériaux, voir Hervé LEUWERS, « Droit des peuples... », op. cit., pp. 215-218 ; voir aussi Marc BELISSA, « Garran de Coulon, la conquête de la Belgique et l'établissement d'un nouveau droit public », Revue du Nord, n 331, 1999, pp. 549-559. 54.Propos tenus au Conseil des Anciens (Le Moniteur, $\mathrm{n}^{\circ} 230$, du 20 floréal an VII, $\mathrm{p}$. 938) ; voir aussi les Réponses de L.M. Révellière-Lépeaux..., op. cit., p. 31.

55.Voir notamment l'article publié dans Le Moniteur, $\mathrm{n}^{\circ} 211$, du 1er floréal an VIII, $\mathrm{p}$. 855.

56. Voir le compte rendu dans Le Moniteur, n 124, du 4 pluviôse an VIII, p. 493.

57. Message du Directoire exécutif au Conseil des Cinq-Cents, reproduit dans Le Moniteur, $\mathrm{n}^{\circ}$ 273, du 3 messidor an VII, réimpression, t. 29, p. 714.

58.Voir notamment L'Examen impartial..., op. cit. (Le Moniteur, $\mathrm{n}^{\circ}$ 124, du 4 pluviôse an VIII, p. 493).

59.Pour reprendre l'expression d'Heurtault-Lamerville. Le Moniteur, $\mathrm{n}^{\circ}$ 262, du 22 prairial an VII, p. 1069. 
60.Fontanes, dans son éloge funèbre de Washington évoque cette région « où semblait commencer une nouvelle époque pour le genre humain ». Au temple de Mars, le 20 pluviôse an VIII (9 février 1800), Le Moniteur, supplément au nº 150, 30 pluviôse, p. 599. 61.Marc BELISSA, « Agrandir le cercle de la civilisation : le débat sur les conséquences de la Révolution américaine », Revue d'histoire moderne et contemporaine, n 46-3, 1999, not. pp. 533-536.

62.Milan, le 17 prairial an VIII (6 juin 1800); Le Moniteur, $\mathrm{n}^{\circ} 265$, du 25 prairial, p. 1669. 63.Procès-verbal de l'installation du gouvernement provisoire du Piémont, du 9 messidor an VIII (28 juin 1800). Le Moniteur, $\mathrm{n}^{\circ}$ 294, du 24 messidor, p. 1185.

64.Oraison funèbre de Washington par Fontanes, le 20 pluviôse an VIII - 9 février 1800 (Le Moniteur, $\mathrm{n}^{\circ}$ 144, du 24 pluviôse, p. 573).

65.Garat évoque aux Anciens, le 16 floréal an VII (5 mai 1799), les « hordes barbares » des Russes : Le Moniteur, ${ }^{\circ}$ 231, du 21 floréal an VII, p. 941.

66.Garat, aux Anciens, le 16 floréal an VII (5 mai 1799), évoque « l'ignorance » des Turcs et des Russes (Le Moniteur, n 231, du 21 floréal an VII, p. 941).

67.Sur l'intérêt qu'ont les princes de l'Empire dans la guerre, Le Moniteur, $\mathrm{n}^{\circ}$ 211, du 1er floréal an VIII, p. 855.

68.Éclaircissements de Talleyrand sur les inculpations qui sont adressées contre lui. Le Moniteur, $\mathrm{n}^{\circ} 299$, du 29 messidor an VII, p. 1216.

69.[Ch. SALADIN], Coup-d'œil politique sur le continent, Paris, C.S. Camus, an VIII-1800, p. 63 (Bib. nat. de France, LB43 81).

70.Article du Morning chronicle du 3 janvier 1800, traduit dans Le Moniteur, $\mathrm{n}^{\circ}$ 117, du 27 nivôse an VIII, p. 463.

71.Sur l'image de Bonaparte : Annie JOURDAN, Napoléon, héros, impérator, mécène, Paris, Aubier, 1998, not. pp. 64-92.

72.Discours du ministre de la Guerre, le 20 pluviôse an VIII (9 février 1800). Le Moniteur, $\mathrm{n}^{\circ}$ 142, du 22 pluviôse an VIII, p. 564.

73.Arch. Nat., AF IV 1449, pièce 119.

74.Ibid., not. pièces 59, 84, 99.

75.Ibid., pièce 97.

76.Ibid., pièce 88.

77.Boris MIRKINE-GUETZEVITCH, «L'influence de la Révolution française sur le développement du droit international dans l'Europe orientale », Recueil des cours de l'Académie de droit international de la Haye, 1928, vol. II, t. 22, p. 317.

78.René MOULINAS, «L'élaboration d'un nouveau droit international : Avignon, le Comtat, et le droit des peuples à disposer d'eux-mêmes (1789-1791) ", dans René MOULINAS, s.dir., La réunion d'Avignon et du Comtat à la France, Avignon, CDDP du Vaucluse, 1992, pp. 45-67. Pour une approche juridique : Jean-François GUILHAUDIS, Le droit des peuples à disposer d'eux-mêmes, Grenoble, P.U.G., 1976, pp.18-19 ; S. CALOGEROPOUlosSTRATIS, Le droit des peuples à disposer d'eux-mêmes, Bruxelles, Émile Bruylant, 1973, p. 11. 79.L'histoire de l'expression est justement rappelée par Jean-Yves GUIOMAR, « Qu'est ce que la Nation? Une définition historique et problématique ", Bulletin de la Société d'histoire moderne et contemporaine, 1996, nos 1-2, pp. 3-4. Sur son usage juridique : S. CALOGEROPOULOS-STRATIS, op. cit. ; Jean-François GUILHAUdis, op. cit. ; Edmond JOUVE, Le droit des peuples, Paris, PUF, 1986, 2e éd., 1992.

80.Edmond JOUve, op. cit., not. pp. 7 et 78-96. 
81.Discours de Petiet, le 15 messidor an VIII (4 juillet 1800). Le Moniteur, $\mathrm{n}^{\circ}$ 295, du 25 messidor, p. 1189.

82.Procès-verbal de la séance générale de la Consulta du Piémont, du 15 messidor an VIII (4 juillet 1800); Le Moniteur, $\mathrm{n}^{\circ} 294$, du 24 messidor, p. 1185.

83.Pour l'an VIII et l'an IX, voir notamment les négociations avec l'Espagne sur le sort du duché de Parme et du nouveau royaume de Toscane (Arch. nat., AF IV 1679) ; pour l'an VII, qu'on se souvienne notamment des bases des négociations de Rastadt : les indemnités territoriales envisagées pour les États allemands dépossédés sur la rive gauche du Rhin, ou les compensations réclamées par la Prusse et l'Autriche (analyse de Jean Debry, du 20 thermidor an VIII dans P. MONTARLOT et L. PINGAUD, op. cit., t. 1, 1912, pp. 121-123).

84.Dénonciation de Ruelle, ancien agent diplomatique, du 23 messidor an VII (11 juillet 1799). Le Moniteur, $\mathrm{n}^{\circ} 324$, du 24 thermidor, p. 1317. Sur cette dénonciation, qui reprenait certaines accusations du Journal des hommes libres, voir Bernard GAINOT, op. cit., p. 109.

85.Dénonciation de Deleschaux, du 25 messidor an VII (13 juillet 1799). Le Moniteur, $\mathrm{n}^{\circ}$ 324, du 24 thermidor an VII, p. 1318.

86.Sur cette politique directoriale dans les Républiques sœurs, voir Jacques GODECHOT, La grande nation, Paris, Aubier, 2e éd., 1983, pp. 357-375.

87.Réponses de L.M. Révellière-Lépeaux..., op. cit., pp. 28-29 ; Bernard NABONNE, op. cit., p. 174 (justification de Reubell).

88. Réponses de L.M. Révellière-Lépeaux..., op. cit., p. 32 ; Ph. Ant. Merlin, membre de l'Institut national..., op. cit., pp. 26-29 ; Bernard NABONNE, op. cit., p. 174 (justification de Reubell). 89.Bernard NABONNE, op. cit., pp. 179-180 (justification de Reubell).

90.Sur ces dernières approches, voir notamment les communications d'Alain-Jacques CZOUZ-TORNARE, de Marita GILLI ou de Jean DELINIÈRE dans Philippe BOURDIN et Bernard GAINOT, s.dir., La République directoriale, Paris, Société des études robespierristes, 1998, t. 2, pp. 961-986, 987-1008, 1011-1024 ; voir aussi Annie JOURDAN, « Les Gaulois en Batavie : des relations diplomatiques machiavéliques », dans Annie JOURDAN et Joep LEERSSEN, s.dir., Remous révolutionnaires : République batave, armée française, Amsterdam, Amsterdam University Press, 1996, pp. 91-117.

91.Pour la période directoriale, nous avions opposé pratiques et théories dans "Théorie et pratiques des relations internationales chez les hommes du Directoire", dans Philippe BOURDIN et Bernard GAINOT, s.dir., op. cit., t. 2, pp. 937-959.

92.Notamment irlandaise. Voir, lors des débats sur l'union de l'Irlande au RoyaumeUni, les positions de Grey, aux Communes, et de lord Holland à la Chambre des pairs (Le Moniteur, $\mathrm{n}^{\circ}$ 222, du 12 floréal an VIII, p. 897 ; id., n² 223, du 13 floréal, p. 901 ; id., n²24, du 14 floréal, p. 905).

93.Gérard MARCOU, « Droits de l'homme et consensus politique », dans Françis HAMON et Jacques LELIÈVRE, s.dir., L'héritage politique de la Révolution française, Lille, PUL, 1993, pp. 23-40. 


\section{RÉSUMÉS}

De part et d'autre du 18 Brumaire, la presse officielle, l'édition et les discours des assemblées diffusent l'image d'un idéal républicain dont l'une des dimensions concerne les relations entre les peuples. La République est présentée comme la gardienne d'un droit des gens, encore fréquemment considéré comme issu du droit naturel; elle prétend rejeter les guerres de conquête, sans pour autant renoncer à de justes compensations; elle assure, enfin, combattre pour la liberté, la civilisation et le respect de la souveraineté des peuples. Au-delà des contradictions évidentes entre discours et pratique, cet article tentera de mettre en évidence la définition et l'évolution de ces principes à un moment clef qui voit la fin de la Révolution proclamée et la guerre de la seconde coalition se poursuivre.

The Republic and Relations between Peoples: Aspects of the Republican tdeal issuing from Brumaire Year VliI. On either side of 18 Brumaire, the official press, publishers and voices in the assemblies spread the image of a Republican Ideal, pertaining, at least in part, to the relations between peoples. The Repubiic was presented as the watchdog of the law of nations, still considered by many to flow from natural law. It purported to reject wars of conquest, without, however, renouncing claims to fair compensation. It further claimed to be fighting for liberty, civilisation and respect for the sovereignty of peoples. Apart from the obvious contradictions between discourse and practice, this article attempts to highlight the ways in which such principles were expressed and evolved at a key moment in time, when the end of the Revolution was proclaimed and the war was resumed with the second coalition.

Repubblica e relazione tra i popoli. Alcuni elementi dell'ideale repubblicano intorno alla data del 18 Brumaio. Da un lato e dall'altro del 18 brumaio la stampa ufficiale, il mondo dell'editoria e i discorsi delle assemblee diffondono l'immagine di un ideale repubblicano, una delle cui dimensioni riguarda le relazioni tra i popoli. La repubblica è presentata come la custode di un diritto delle genti, encore frequentemente considerato come derivato dal diritto naturale; essa pretende di rifiutare le guerre di conquista, senza per questo rinunciare a dei giusti indennizzi; essa assicura, infine, di combattere per le libertà, la civiltà e il rispetto della sovranità dei popoli. Al di là delle contraddizioni evidenti tra discorsi e pratica, quest'articolo tenterà di evidenziare la definizione e l'evoluzione di questi principi in un momento chiave, che vede proclamata la fine della Rivoluzione, mentre prosegue la guerra della seconda coalizione.

Antes y despues del 18 brumaire, la prensa oficial, la edición y los discursos de las asembleas difunden la imagen de un ideal republicano que tiene como una de sus dimensiones las relaciones entre los pueblos. La República esta presentada como la salvaguarda del derecho de la gente, concebido todavía como procedente del derecho natural. Pretende rechazar las guerras de conquista, sin renunciar sin embargo a compensaciones justas, asegura por fín combatir por la libertad, la civilización y la soberanía de los pueblos. Más allá de las contradicciones claras entre discurso y práctica, este artículo intenta definir esos principios y sus evoluciones en un momento clave: el final de la revolución proclamada y la continuación de la guerra de la segunda coalición.

Die französische Marine am 18. Brumaire. Kurz vor dem 18. Brumaire scheint die französische Kriegsmarine in einem jämmerlichen Zustand zu sein. Die Finanzschwierigkeiten erlauben nicht mehr, Schiffe auszurüsten, Matrosen und Arbeiter bekommen keinen Lohn mehr. Die Aushebungen sind unzureichend und die Desertionen zahlreich. Die atlantischen Häfen sind wegen der Unruhen, die im Hinterland herrschen, isoliert. Die offiziellen Zeremonien zeugen immerhin von Treue zu der Regierung, wenigstens von einer gewissen Anpassung. Soweit man 
es beurteilen kann, wird der Staatsstreich ziemlich positiv aufgenommen. Aber die darauf folgenden Zeremonien und die Vereidigungen zeigen eine wachsende Militarisierung und eine mehr oder weniger erzwungene Einstimmigkeit.

\section{AUTEUR}

HERVÉ LEUWERS

Université Lille III - Charles de Gaulle 